\title{
An Adaptive Multiscale AM-FM Texture Analysis System With Application to Hysteroscopy Imaging
}

\author{
Ioannis Constantinou*, Marios Pattichis $\#$, Vasilis Tanos ${ }^{\Uparrow}$, Marios Neofytou*, Constantinos Pattichis* \\ *Department of Computer Science, University of Cyprus Nicosia, Cyprus \\ ${ }^{\#}$ Department of Electrical and Computer Engineering, The University of New Mexico, Albuquerque, NM, USA \\ "Aretaeion Medical Center, Nicosia, Cyprus
}

\begin{abstract}
The use of multiscale AM-FM analysis systems has been recently demonstrated in a variety of applications in medical image analysis. In all of these applications, a fixed filter-bank is used as a preprocessing step for estimating different AM-FM components from different scales. In this paper, for the first time, we introduce the use of an adaptive, multiscale AM-FM approach that searches for the optimal filter-bank specification for use in image classification. We demonstrate an example application in hysteroscopy imaging, for identification of gynaecological cancer, where the optimal filter-bank turns out to be circularly symmetric.
\end{abstract}

Keywords - multiscale medical imaging analysis, AM-FM representation, cancer, hysteroscopy imaging.

\section{INTRODUCTION}

Multiscale AM-FM methods have been used in a wide range of medical imaging analysis applications and in computer aided diagnosis systems (CADs) Error! Reference source not found.. Recent applications have been documented in ophthalmology [1], in ultrasound images, in magnetic resonance imaging [4] and nuclear medicine [4].

The main advantage of AM-FM representation is that the AM-FM decompositions provide physically meaningful image texture measurements. Significant texture variations are well represented in the frequency components. For single component FM cases, Instantaneous-Frequency (IF) vectors are orthogonal to equi-intensity lines of an image, while the IF magnitude provides a measure of local frequency content. By using AM-FM components from different scales, we can produce multiscale features at pixel-level resolutions [5].

Since AM-FM texture features are provided at a pixel level resolution, AM-FM models can be used to segment texture images that are difficult to model with the standard brightness-based methods [6]. On the other hand, using just histograms of the IF and Instantaneous Amplitude (IA), we can design effective content-based image retrieval systems using very short image feature vectors [1].

In the past few years, several medical applications based on AM-FM methods were developed. Boudraa et al. [4] introduced a new cross-energy operator and used the operator to demonstrate the functional segmentation of dynamic nuclear images. Maragos et al. [7] provide an important application for ultrasound spectroscopy in [7], where AM-FM models are used for improving Doppler ultrasound resolution. Vector-valued based AM-FM demodulation is given in [8] by Alexandratou et al. Multiscale AM-FM methods were applied to chest radiographs [9], ultrasound images of the carotid artery [10], classification of surface electromyographic signals [11], and retinal image classification [1].

In this paper, we introduce an adaptive multiscale AM-FM decomposition system that can be adapted to different applications. The significant advantages of the proposed approach are demonstrated in an application in hysteroscopy imaging.

Hysteroscopy examination is considered to be the gold-standard technique for the diagnosis of intrauterine pathology [12]. Within the female population, gynaecological cancer accounts for the second highest mortality rate. Early diagnosis and treatment of gynaecological cancer are essential for better quality and longer life.

The rest of the paper is organized in the following three sections. Method include the fundamentals of AMFM, the representation of adaptive multiscale AM-FM texture analysis system, and a medical application to hysteroscopic imaging, the results represent the results of the medical application and the last section including the discussion remarks.

\section{MethodOLOGY}

\section{A. AM-FM theory}

Non-stationary image content is described using a multiscale AM-FM representation using:

$$
\mathrm{I}(x, y)=\sum_{n=1}^{M} \mathrm{a}_{\mathrm{n}}(\mathrm{x}, \mathrm{y}) \cos \varphi_{n}(x, y),
$$

where $\mathrm{n}=1,2 \ldots M$ denote different frequency scales, $\mathrm{a}_{\mathrm{n}}(\mathrm{x}, \mathrm{y})$ denote the Instantaneous Amplitude (IA) functions, and $\varphi_{n}(x, y)$ denote the instantaneous phase (IP) functions. For each phase function $\varphi_{n}(x, y)$, we define the instantaneous frequency (IF) $\nabla \varphi_{n}(x, y)$ in terms of the gradient:

$$
\nabla \varphi_{n}(x, y)=\left(\frac{\partial \varphi_{n}(x, y)}{\partial x}, \frac{\partial \varphi_{n}(x, y)}{\partial y}\right)
$$

Each scale is defined in terms of a collection of band pass filters that share similar magnitude range. 


\section{B. AM-FM Demodulation}

AM-FM demodulation methods are used for estimating the IA, IP and IF for each AM-FM component. In this paper, AM-FM demodulation based on the multiscale approach introduced in [5].

To summarize the approach, let $I(x, y)$ be the input image. A 2D extension of the analytic signal is computed using:

$$
I_{A S}(x, y)=I(x, y)+j H_{2 d}[I(x, y)],
$$

where $H_{2 d}$ denotes 2D Hilbert transform operator (1D Hilbert operator applied along the rows or columns). The $2 \mathrm{D}$ analytic signal is then processed through a filterbank. For each filterbank output, $I_{A S_{n}}$, the IA, IP, and IF estimates are obtained using:

$$
\begin{aligned}
& \alpha_{n}(x, y) \approx\left\|I_{A S_{n}}(x, y)\right\|, \text { and } \\
& \varphi_{n}(x, y) \approx \arctan \left(\frac{\operatorname{imag}\left(I_{A S_{n}}(x, y)\right)}{\operatorname{real}\left(I_{A S_{n}}(x, y)\right)}\right) .
\end{aligned}
$$

Estimation of the IF components requires the use of inverse trigonometric functions. Because of the numerical instability associated with evaluating inverse trigonometric functions, the VS-LLP was proposed in [5] to provide more accurate estimates. For the $x$-component, the VS-LLP selects the best estimate among

$$
\frac{\partial \varphi}{\partial x}(x, y) \approx \frac{1}{n_{1}} \arccos \left(\frac{I_{A S}\left(x+n_{1}, y\right)+I_{A S}\left(x-n_{1}, y\right)}{2 I_{A S}(x, y)}\right)
$$

for integer values of $n_{1}$. The procedure is repeated for the $y$-component.

\section{Adaptive multiscale AM-FM texture analysis system}

The estimated multiscale AM-FM features can be greatly affected by the choice of scales. In term, these scales are defined in terms of the underlying filterbank. We propose an adaptive, multiscale AM-FM approach that estimates the optimal filterbank configuration based on the targeted application.

\begin{tabular}{cl}
\hline & Preprocessing \\
1 & Acquire texture image \\
2 & Extract ROI from image \\
3 & Convert ROI to the YCrCb color space \\
\hline \multicolumn{3}{c}{ Multiscale AM-FM analysis with different filterbanks } \\
4 & Apply 2D Hilbert transform to each region of interest \\
6 & Select filterbank parameters from given set \\
7 & Generate filterbank \\
& Estimate IA, IP, and IF. \\
8 & Performance evaluation: \\
9 & Estimate performance \\
10 & Save results \\
\hline & Return to step 4 until all filterbanks have been considered \\
11 & Select optimal filterbank configuration \\
12 & Implement AM-FM system with optimal filterbank
\end{tabular}

Figure 1. Adaptive multiscale AM - FM framework for texture analysis
The adaption of the filterbank is key in automating AM-FM image analysis for optimal performance on any given application. Figure 1 summarizes the steps of the proposed AM-FM texture analysis system.

Preprocessing: This stage includes image acquisition, ROI extraction and color conversion. ROI extraction can be performed manually, semi-automatically, or automatically.

Multiscale AM-FM analysis with different filterbanks: As we shall explain below, filterbanks are parameterized in terms of the number of scales and orientations. In this paper, a filterbank is specified in the frequency-domain. Performance evaluation can be measured differently depending on the application. For each filter-bank, for the purposes of this paper, the classification performance is evaluated.

The collection of filter-banks that are being considered is summarized in Table I. To make the problem tractable, we only consider scales that are defined in terms of frequency-radii that are multiples of $\pi / 16$. We consider 1 , 2 , or 4 possible orientations. Overall, filter-bank definition depends on 6 variables: overlap, number of orientations, dominant filter selection mode (all or multi-scale), and the number of scales (in case of 4 scale definition we need 3 variables).

TABLE I. Set Of Filter-Banks That Were Considered.

\begin{tabular}{lll}
\hline & Parameters & Possible values \\
\hline 1 & Overlap & 0 or $25 \%$ \\
2 & Mode & All or Multiscale \\
3 & Number of & 1,2 or 4 \\
& Orientations & $1 / 16 \pi, 2 / 16 \pi, 3 / 16 \pi, 4 / 16 \pi, 5 / 16 \pi$, \\
& & $6 / 16 \pi, 7 / 16 \pi, 8 / 16 \pi, 9 / 16 \pi, 10 / 16 \pi$, \\
4 & Scale $1: \mathrm{R}_{1}$ & $11 / 16 \pi, 12 / 16 \pi$ \\
& & $2 / 16 \pi, 3 / 16 \pi, 4 / 16 \pi, 5 / 16 \pi, 6 / 16 \pi$, \\
& & $7 / 16 \pi, 8 / 16 \pi, 9 / 16 \pi, 10 / 16 \pi, 11 / 16 \pi$, \\
5 & Scale $2: \mathrm{R}_{2}$ & $12 / 16 \pi, 13 / 16 \pi$ \\
& & $3 / 16 \pi, 4 / 16 \pi, 5 / 16 \pi, 6 / 16 \pi, 7 / 16 \pi$, \\
& & $8 / 16 \pi, 9 / 16 \pi, 10 / 16 \pi, 11 / 16 \pi, 12 / 16 \pi$, \\
6 & Scale $3: \mathrm{R}_{3}$ & $13 / 16 \pi, 14 / 16 \pi$ \\
& &
\end{tabular}

Exhaustive search in the space of all possible filterbanks requires

$$
2 * 2 * 3 *\left(\begin{array}{c}
15 \\
3
\end{array}\right)=20,736
$$

different possibilities.

Select Optimal Filterbank Configuration: In the last step, the filter-bank that gives the optimal performance is selected. This filter-bank is then implemented.

\section{Medical Application}

The proposed Adaptive Multiscale AM-FM texture analysis system was applied to the assessment of endometrial cancer. The system implementation requires the use of an upgraded version of the hysteroscopic standardized protocol that was documented in [13] Preprocessing: This stage includes hysteroscopy image 
acquisition, semiautomatic ROI extraction and ROI color conversion.

Multiscale AM-FM analysis with different filterbanks: For each filter-bank, the IA, IP and IF estimates were extracted from different scales. Then for each AM-FM component estimate, we calculate the entropy, the median value, and the Inter-Quartile Range (IQR). For comparing between normal and abnormal cases, significant difference between feature sets is evaluated using the Wilcoxon Rank Sum statistical test. AM-FM features that do not show a significant difference are discarded from further consideration. The overall classification of each filter-bank is based on the classification score.

\section{RESULTS}

\section{A. Medical Application}

The Adaptive Multiscale AM-FM Texture Analysis system was applied to hysteroscopy images for the assessment of endometrial cancer. The derived AM-FM features were input into an SVM classifier. From a total of 200 ROIs, we present averages over 20 experiments of training over 50 random samples and testing over another set of 50 random samples. A total of 20,737 filter-banks were investigated. The highest correct classification score was $\mathrm{CC}=82,8 \%$. A summary of the results is given in Table II.

TABLE II. Summary Of The Best CORReCt Classification SCORE (\%CC) FOR DiFFERENT NUMBER OF ORIENTATIONS.

\begin{tabular}{ccccccc}
\hline $\begin{array}{c}\text { Number of } \\
\text { Orientations }\end{array}$ & Mode & \%Overl. & $R_{s 1}$ & $R_{s 2}$ & $R_{s 3}$ & \%CC \\
\hline 1 & multi & $12,5 \%$ & $\pi / 16$ & $5 \pi / 16$ & $12 \pi / 16$ & 82,8 \\
1 & all & $12,5 \%$ & $\pi / 16$ & $7 \pi / 16$ & $15 \pi / 16$ & 82,7 \\
2 & all & $12,5 \%$ & $8 \pi / 16$ & $14 \pi / 16$ & $15 \pi / 16$ & 81,0 \\
2 & multi & $0 \%$ & $8 \pi / 16$ & $11 \pi / 16$ & $14 \pi / 16$ & 80,4 \\
4 & all & $0 \%$ & $\pi / 16$ & $3 \pi / 16$ & $4 \pi / 16$ & 72,8 \\
4 & all & $0 \%$ & $2 \pi / 16$ & $4 \pi / 16$ & $8 \pi / 16$ & 82,8 \\
\hline
\end{tabular}

In the results, note that the case of " 1 " for the number of orientations refers to the fact that the entire scale was covered by a single filter (bandpass or donut-like filter). This turned out to be the best case and it reflects the case of perfect circular symmetry. Furthermore, in terms of classification by scale, the low and medium frequency scales gave the best classification results. For the optimal scale specification, a more detailed presentation of the results is given in Table III. It is interesting to note that the use of several orientations requires more overlap to rival the performance of the circularly symmetric cases. In other words, the approximation to the circularly symmetric filter-bank determines how close we get to the best results.

In table IV, we summarize the significant differences that were found between normal and abnormal cases. It is clear from the table, that all AM-FM features show significant difference except for the Low frequency IA.
TABLE III. Classification Performance For The Optimal SCALE Specification. For All Cases, We Are Using The Multiscale Mode That Selects The Optimal Per Scale.

\begin{tabular}{|c|c|c|c|c|c|}
\hline Scale & $\begin{array}{c}\text { Num. } \\
\text { of } \\
\text { Orns*. }\end{array}$ & $\begin{array}{c}\% \\
\text { Overlap }\end{array}$ & $\begin{array}{c}\% \\
\mathrm{CC}\end{array}$ & Sensitivity & Specificity \\
\hline \multirow{6}{*}{$0-\pi / 16$} & 1 & 0 & 82 & 76 & 88 \\
\hline & 1 & 25 & 80 & 77 & 82 \\
\hline & 2 & 0 & 81 & 75 & 87 \\
\hline & 2 & 25 & 81 & 76 & 86 \\
\hline & 4 & 0 & 82 & 77 & 87 \\
\hline & 4 & 25 & 82 & 74 & 90 \\
\hline \multirow{6}{*}{$\begin{array}{l}\pi / 16- \\
6 \pi / 16\end{array}$} & 1 & 0 & 81 & 76 & 85 \\
\hline & 1 & 25 & 81 & 77 & 86 \\
\hline & 2 & 0 & 82 & 75 & 90 \\
\hline & 2 & 25 & 79 & 72 & 87 \\
\hline & 4 & 0 & 81 & 76 & 86 \\
\hline & 4 & 25 & 81 & 78 & 85 \\
\hline \multirow{6}{*}{$\begin{array}{l}6 \pi / 16- \\
12 \pi / 16\end{array}$} & 1 & 0 & 75 & 66 & 84 \\
\hline & 1 & 25 & 72 & 66 & 78 \\
\hline & 2 & 0 & 74 & 73 & 75 \\
\hline & 2 & 25 & 77 & 73 & 82 \\
\hline & 4 & 0 & 70 & 64 & 76 \\
\hline & 4 & 25 & 77 & 69 & 85 \\
\hline \multirow{6}{*}{$\begin{array}{c}12 \pi / 16- \\
16 \pi / 16\end{array}$} & 1 & 0 & 71 & 68 & 74 \\
\hline & 1 & 25 & 77 & 78 & 77 \\
\hline & 2 & 0 & 69 & 65 & 74 \\
\hline & 2 & 25 & 67 & 61 & 72 \\
\hline & 4 & 0 & 69 & 68 & 70 \\
\hline & 4 & 25 & 76 & 75 & 78 \\
\hline
\end{tabular}

TABLE IV. WiLKoxon RANK SUm STATISTical Test BetweEn AMFM FEATURE EXTRACTED From NORMAL AND ABNORMAL ROIS. A VALUE OF ' 1 ' SUGGEST SigNIFICANT DIFFERENCE.

\begin{tabular}{lccc}
\hline & \multicolumn{3}{c}{ Texture Feature } \\
\cline { 2 - 4 } Component & Entropy & Median & $I Q R$ \\
\hline Low IA & 0 & 1 & 1 \\
Low IFx & 1 & 1 & 1 \\
Low IFy & 1 & 1 & 1 \\
\hline Medium IA & 1 & 1 & 1 \\
Medium IFx & 1 & 1 & 1 \\
Medium IFy & 1 & 1 & 1 \\
\hline
\end{tabular}

In Fig. 2, we present an example AM-FM component for the medium frequency scales using a circularlysymmetric filter-bank. It is interesting to note that the ROI features are significantly amplified in the medium IA and FM $(\cos (\varphi))$. Furthermore, there is considerably more variation in the AM-FM features associated with the abnormal ROI as compared to the features associated with the normal ROI. We also note that these strong variations are captured by the use of a wide bandwidth for the medium-frequency scale that ranges from a radius of $\pi / 16$ to $6 \pi / 16$ in the discrete frequency plane.

\section{DISCUSSION}

An Adaptive Multi-scale AM-FM texture analysis system has been developed in this paper. The method provides for a general framework for evaluating a large number of filter-bank designs. The results indicate that a circularly-symmetric filter-bank gave the best results. Further the results are promising more robust 
classification score in comparison with the classical contrast based texture analysis [13].

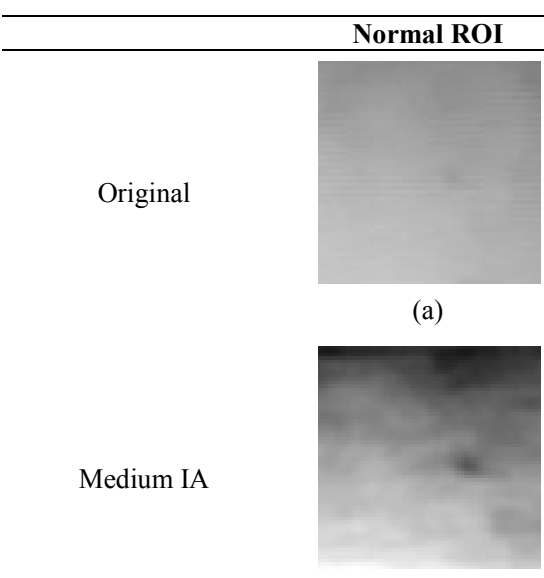

(c)

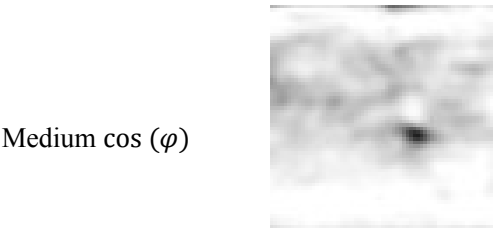

(e)

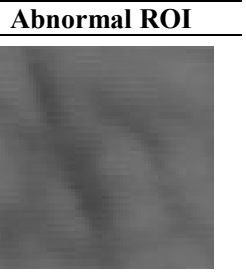

(b)

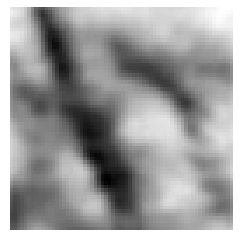

(d)

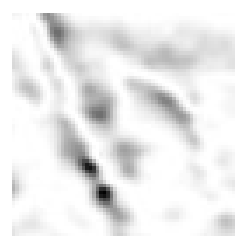

(f)
Figure 2. Medium-frequency scale decomposition for normal and abnormal ROIs. The normal ROI is shown in (a). The abnormal ROI is shown in (b). The Medium IAs is shown in (c) and (d). The Medium FM images are shown in (e) and (f).

\section{REFERENCES}

[1] V. Murray, M. S. Pattichis, E. Barriga, and P. Soliz, "Recent multiscale AM-FM methods in emerging applications in medical imaging," EURASIP Journal on Advances in Signal Processing, vol. 2012, no. 1, p. 23, 2012.

[2] C. Agurto, V. Murray, E. Barriga, S. Murillo, M. S. Pattichis, H. Davis, S. Russell, M. Abramoff, and P. Soliz, "Multi-scale AMFM methods for diabetic retinopathy lesion detection", IEEE Trans. Med. Imag.,vol. 29, (2), pp. 502-512, 2010.

[3] C. P. Loizou, V. Murray, M. S. Pattichis, I. Seimenis, M. Pantziaris, and C.S. Pattichis, "Multiscale Amplitude-Modulation Frequency-Modulation (AM-FM) Texture Analysis of Multiple Sclerosis in Brain MRI Images", IEEE Trans. Inf. Technol. Biomed., vol. 15, (1), pp. 119-129, 2011.

[4] A. O. Boudraa, J. C. Cexus, H. Zaidi, "Functional segmentation of dynamic nuclear images by cross-ifb-energy operator", Comput. Methods Progr. Biomed, vol. 84, (2-3), pp. 146-152, 2006.

[5] V. Murray, P. Rodriguez, and M. Pattichis, "Multiscale AM-FM demodulation and image reconstruction methods with improved accuracy", IEEE Trans. Image. Process., vol. 19, (5), pp. 1138 1152, 2010.

[6] M. S. Pattichis, C. Pattichis, M. Avraam, A. Bovik, K. Kyriacou, "AM-FM texture segmentation in electron microscopic muscle imaging”, IEEE Trans. Med. Imag., vol 19, (12), pp. 1253-1257, 2000 .
[7] P. Maragos, T. Loupas, V. Pitsikalis, "Improving doppler ultrasound spectroscopy with multiband instantaneous energy separation", in Proc $14^{\text {th }}$ International Conference on Digital Signal Processing, 2002, vol. 2, pp. 611-614.

[8] E. Alexandratou, A. Sofou, H. Papasaika, P. Maragos, D. Yova, N. Kavantzas, "Computer vision algorithms in DNA ploidy image analysis", in Proc. of the SPIE Imaging, Manipulation, and Analysis of Biomolecules, Celss, and Tissues, ed. by Farkas DL, Nicolau DV, Leif RC, 2006, vol. 6088, pp. 180-190.

[9] V. Murray, M. S. Pattichis, H. Davis, E. S. Barriga, P Soliz, "Multiscale AM-FM analysis of pneumoconiosis x-ray images", IEEE International Conference on Image Processing, 2009, pp. 4201-4204.

[10] C. Loizou, V. Murray, M. S. Pattichis, M. Pantziaris, and C. S. Pattichis, "Multi-scale amplitude modulation-frequency modulation (am-fm) texture analysis of ultrasound images of the intima and media layers of the carotid artery", IEEE Trans Inf. Tech Biomed., vol. 15, (2), pp. 178-188, 2011.

[11] C. I. Christodoulou, P. A. Kaplanis, V. Murray, M. S. Pattichis, C. S. Pattichis and T. Kyriakides, "Multi-scale AM-FM analysis for the classification of surface electromyographic signals", Biomedical Signal Processing and Control, vol. 7, (3), pp. 254$269,2012$.

[12] I. P. Constantinou, M. S. Neofytou, V. Tanos, and C. S. Pattichis, "Reliable Hysteroscopy Color Imaging", The 12th Mediterranean Conference on Medical and Biological Engineering and Computing, 2010, pp. 201-206.

[13] I. P. Constantinou, C. A. Koumourou, M. S. Neophytou, V. Tanos, C. S. Pattichis, and E. C. Kyriacou, "An Integrated CAD System Facilitating the Endometrial Cancer Diagnosis", CD-ROM Proceedings of the 9th International Conference on Information Technology and Applications in Biomedicine, ITAB 2009, Larnaca, Cyprus, 5 pages, Nov. 5-7, 2009. 